\title{
Analyse de Cycle de Vie d'un bâtiment
}

\author{
Maxime Trocmé ${ }^{1}$, Bruno Peuportier ${ }^{2}$ \\ ${ }^{1}$ Vinci Construction France, Direction Recherche \& Développement, 61 avenue Jules Quentin, 92730 Nanterre Cedex - Tel : 01.46 .95 .76 .83 - email : \\ mtrocme@gtm-construction.com \\ ${ }^{2}$ Ecole des Mines de Paris, Centre Energie et Procédés, 60 Bd St Michel, 75272 Paris Cedex 06 - Tel : 01.40 .51 .91 .51 - email : bruno.peuportier@ensmp.fr
}

Préambule - Cet article, avant relecture et correction pour la revue J3EA, a fait l'objet d'une communication aux journées 2007 de la section électrotechnique sur le thème Energie et Développement Durable à l'antenne de Bretagne de l'ENS de Cachan les 14 et 15 mars 2007.

Résumé-La méthode d'Analyse de Cycle de Vie du bâtiment développée au Centre Energétique et Procédés de l'Ecole des Mines de Paris permet d'évaluer les principaux impacts environnementaux (effet de serre, eutrophisation, consommation d'eau...) d'un bâtiment issus de sa construction, son utilisation, sa rénovation et sa destruction.

Cette démarche, résultat du chaînage entre les logiciels ALCYONE, COMFIE et EQUER, est appliquée aux bâtiments du quartier Lyon Confluence.

Différentes variantes sont proposées pour situer le niveau de performances du projet par rapport à la réglementation et aux meilleures pratiques.

\section{INTRODUCTION}

Les bâtiments sont souvent pointés du doigt dans le cadre de la lutte contre le changement climatique. Ils contribuent ainsi à $23 \%$ des émissions directes de gaz à effet de serre [1] et $47 \%$ des consommations d'énergie finale [2]. Ils sont aussi à l'origine d'autres conséquences environnementales semblant pouvoir compromettre la capacité des générations futures à répondre à leurs besoins (épuisement des ressources, eutrophisation des rivières, acidification des pluies, production de déchets radioactifs...).

L'éco-conception d'un bâtiment consiste en la prise en compte de ces paramètres, pas uniquement en son optimisation énergétique. Cette dernière est primordiale dans la conception d'un "bâtiment durable" mais ne reflète pas les impacts environnementaux dus à la construction, la démolition ou encore au choix du type d'énergie.

La démarche et les logiciels présentés ici constituent un outil d'aide à la décision. Par une quantification précise des impacts environnementaux, ils permettent aux acteurs de la construction de faire le choix le plus cohérent par rapport à leurs objectifs.

\section{Methodologie}

\section{A. Analyse de Cycle de Vie}

L'Analyse de Cycle de Vie (ACV) permet d'évaluer les impacts environnementaux d'un produit, depuis sa fabrication jusqu'à sa fin de vie. Cette méthode a été utilisée par différents fabricants de matériaux de construction et plusieurs outils sont proposés pour les bâtiments [3].

\section{B. Modélisation d'un bâtiment}

Un bâtiment possède deux types de frontières. On peut d'abord évoquer la frontière «physique » qui comprend tous ses éléments. On parle aussi de frontière «flux» qui doit contenir les procédés amont (production d'énergie, traitement des eaux, fabrication et transport des matériaux de construction) et aval (gestion des déchets, des eaux usées...) [4]. La définition de cette frontière permet au système d'interagir avec l'environnement extérieur via des flux élémentaires :

- Flux de ressources : eau, énergie, matières premières ;

- Emissions : gazeuses, liquides, solides.

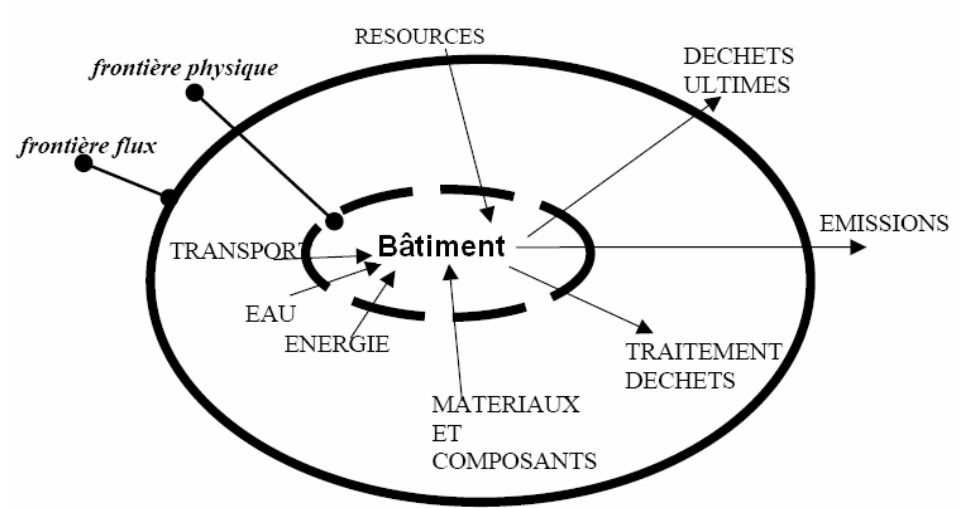

Fig. 1. Frontières du système étudié

Selon la méthodologie de l'ACV [5], les substances puisées et émises dans l'environnement sont comptabilisées (phase d'inventaire) puis des indicateurs environnementaux sont déduits pour les quatre phases du cycle de vie du quartier :

- Construction : extraction des matières premières, production et transport des matériaux ;

- Utilisation: chauffage, éventuellement 
climatisation, consommation d'eau...;

- Rénovation: remplacement des composants (fenêtres, revêtements des bâtiments et des rues) ;

- Démolition : transport et traitement des déchets.

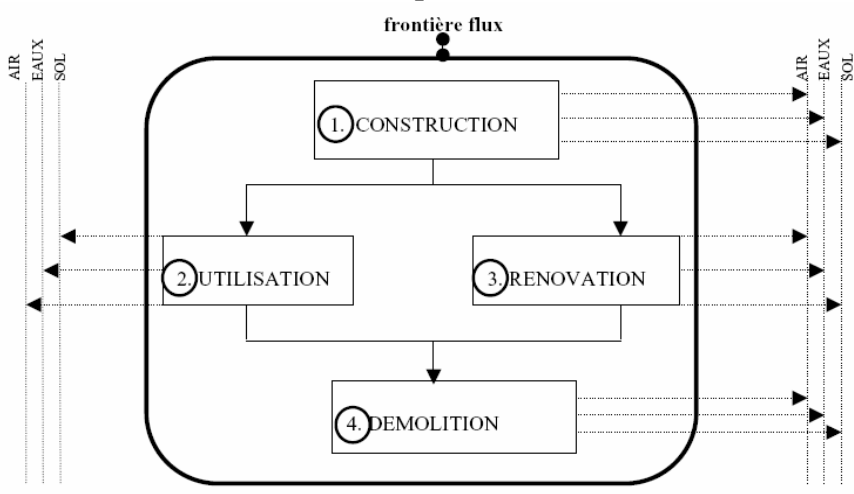

Fig. 2. Modélisation de l'ACV d'un bâtiment

Il convient également de tenir compte des aspects liés au comportement des résidents (consommation d'eau et d'énergie, traitement des déchets, pourcentage de tri et de recyclage, etc.) et des caractéristiques du site (distances de transport, climat, énergies utilisées pour la production d'électricité et de chauffage urbain, etc.).

La simulation du cycle de vie est effectuée sur une période d'analyse, qui peut correspondre à la durée de vie supposée d'un bâtiment (par exemple 80 ans), en utilisant un pas de temps annuel.

\section{Indicateurs considérés}

Les données environnementales concernant la fabrication des matériaux et les différents procédés inclus dans le système (production d'énergie et d'eau, traitement des déchets et des eaux usées, transports...) sont issues de la base Ecoinvent développée par des instituts de recherche suisses [6]. Cette base fournit pour chaque procédé et matériau, en fonction de l'unité de référence considérée (par exemple $\mathrm{kg}$ pour les matériaux, TJ pour l'énergie, tonne-km pour le transport des marchandises), un inventaire de cycle de vie c'est à dire l'ensemble des flux de matière et d'énergie entrant et sortant du système :

- Ressources utilisées: matériaux rares, eau, énergie ;

- Emissions dans l'air, l'eau et le sol : CO2 dans l'air, ammoniaque dans l'eau, métaux dans le sol...;

- Déchets créés : déchets inertes, toxiques, radioactifs...

Des indicateurs environnementaux sont alors évalués (cf. Tableau 1), par exemple la contribution à l'effet de serre est calculée en fonction des quantités de chaque gaz (données dans l'inventaire), pondérées par le potentiel de réchauffement global correspondant [7].

Ces indicateurs sont évalués pour le bâtiment sur son cycle de vie. En utilisant un diagramme radar comportant 12 axes associés à ces 12 indicateurs, diverses alternatives peuvent être comparées pour améliorer le projet du point de vue environnemental.

\begin{tabular}{ll}
\hline \multicolumn{1}{c}{ Indicateur environnemental } & \multicolumn{1}{c}{ Unité } \\
\hline Demande cumulative d'énergie [6] & $\mathrm{GJ}$ \\
Eau utilisée [6] & $\mathrm{m}^{3}$ \\
Epuisement des ressources abiotiques [8] & $\mathrm{kg}$ antimoine eq. \\
Déchets produits [6] & $\mathrm{t}$ \\
Déchets radioactifs [6] & $\mathrm{dm}^{3}$ \\
Effet de serre (100 ans) [8] & $\mathrm{t} \mathrm{CO}_{2}$ eq. \\
Acidification [8] & $\mathrm{kg} \mathrm{SO}_{2}$ eq. \\
Eutrophisation [8] & $\mathrm{kgPO}_{4}{ }^{3-}$ eq. \\
Dommage à la qualité des écosystèmes dus à l'écotoxicité [9] $\mathrm{PDF}^{2} \mathrm{~m}^{2}$ \\
Dommage à la santé [9] & $\mathrm{DALY}^{2}$ \\
Production d'ozone photochimique [8] & $\mathrm{kg} \mathrm{C}_{2} \mathrm{H}_{4}$ eq. \\
Odeur [8] & $\mathrm{m}^{3}$ air \\
\hline
\end{tabular}

TABLEAU 1 : LES INDICATEURS ENVIRONNEMENTAUX EVALUES

Les indicateurs sont également présentés en montrant la contribution relative de chaque phase principale du cycle de vie (construction, utilisation, rénovation, et démolition). Ceci permet de mieux cerner les possibilités d'améliorer un projet : par exemple si l'impact de la phase d'utilisation est trois fois plus grand que celui de la construction, la priorité peut être donnée aux économies de flux (énergie, eau, déchets) plutôt qu'à l'utilisation de matériaux à moindre impact.

\section{OUTILS INFORMATIQUE}

L'analyse de cycle de vie d'un bâtiment est le résultat d'un chaînage de données entre différents outils informatiques d'évaluation :

- ALCYONE: description géométrique du bâtiment [10] ;

- COMFIE : simulation thermique [11] ;

- EQUER : ACV des bâtiments [12].

Ces outils sont chaînés via des fichiers textes dans lesquels les données de bâtiment (la géométrie, les besoins d'énergie, les quantités et types de matériaux impliqués) sont écrites par l'outil situé en amont et lues par l'outil situé en aval.

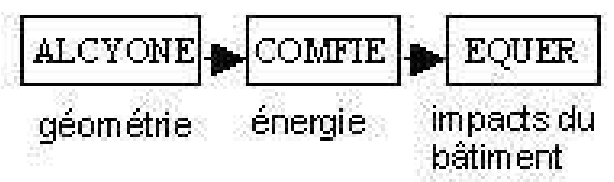

Figure 3: Chaînage des entrées/sorties entre les outils d'évaluation

ALCYONE - le modeleur 2d-3d transfère les données architecturales à l'outil de simulation thermique COMFIE. Le bâtiment est décomposé en volumes appelés zones ayant un comportement thermique homogène (température unique).

COMFIE - un modèle est importé d'ALCYONE, complété par des données sur l'utilisation du bâtiment, puis la simulation est effectuée en utilisant des données météorologiques horaires. Le programme calcule les besoins d'énergie (chauffage, climatisation et éclairage) et des températures horaires pour les différentes zones thermiques du bâtiment. Les données de l'enveloppe, définie par ses 
matériaux et leurs quantités, et les besoins d'énergie, sont alors transférés à l'outil EQUER.

EQUER - un modèle est importé de COMFIE, complété par des données sur les déchets, les consommations d'eau, éventuellement les transports domicile-travail, puis l'analyse de cycle de vie est effectuée pour évaluer le profil environnemental du bâtiment.

\section{APPLICATION A LYON CONFLUENCE}

\section{A. Présentation}

Le projet Lyon Confluence concerne la requalification de l'ensemble de la Presqu'île entre le Rhône et la Saône au delà de la gare SNCF de Perrache, soit près de 150 ha. La zone d'étude sélectionnée (îlots A, B et C, cf. Figure 2) représente environ $60000 \mathrm{~m}^{2}$ de logements et $15000 \mathrm{~m}^{2}$ de bureaux. En appliquant le modèle décrit précédemment, les performances $\mathrm{du}$ projet sont comparées à deux références, l'une correspondant au standard actuel et l'autre aux meilleures pratiques : trois variantes sont donc définies.

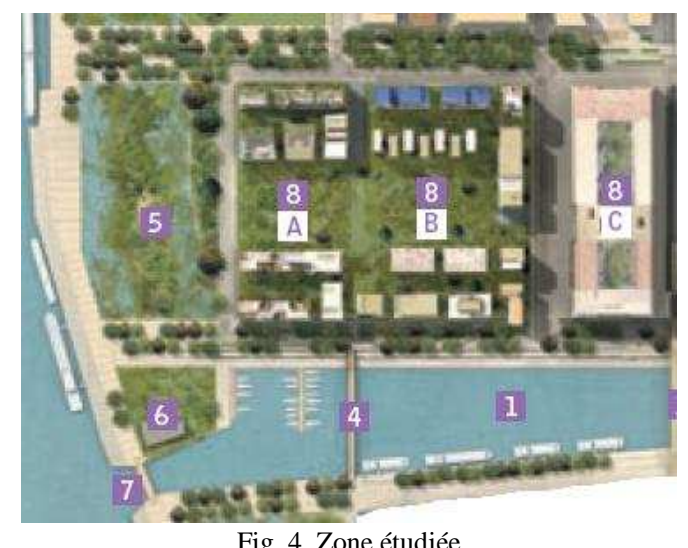

La variante Standard correspond environ aux niveaux d'exigences réglementaires en construction neuve (RT2005 et techniques communément mises en œuvre en France).

La variante Base correspond aux choix techniques et architecturaux retenus par la SEM Lyon Confluence pour répondre aux objectifs du programme européen Concerto : $40 \%$ de consommation énergétique par rapport à la RT2000, prise en charge par les ENR de $80 \%$ des besoins de chauffage et ECS, et de $50 \%$ des consommations électriques des espaces communs).

La variante Meilleures Pratiques correspond aux techniques existantes les plus performantes (généralisation de la ventilation double flux, sur-isolation, traitement des ponts thermiques).

\section{B. Choix techniques innovants}

Les concepteurs des bâtiments ont mis en œuvre différentes techniques permettant d'atteindre les objectifs de Concerto.

Les constructions étudiées ici montrent plusieurs approches pour optimiser les performances de l'enveloppe. D'abord, la plupart des bâtiments considérés présentent une bonne compacité (rapport surface d'enveloppe sur surface d'étage). Plus le bâtiment sera compact moins ses déperditions par les parois seront importantes. Ensuite, les concepteurs ont pris en compte la problématique des apports solaires (solaire passif). Les vitrages au Sud sont privilégiés par rapport aux autres orientations, ce qui permet de capter le maximum d'énergie en hiver. Pour répondre à la problématique du confort d'été, des brises soleils permettront de minimiser ces apports.

L'enveloppe des bâtiments est sur-isolée. Plus l'épaisseur d'isolant est importante plus la résistance thermique des parois le sera. Le tableau 2 illustre l'épaisseur d'isolant pour les variantes considérées.

Certains bâtiments présentent une isolation intérieure (technique communément mise en œuvre en France). Ce type d'isolation a pour inconvénient de présenter des ponts thermiques. Les ponts thermiques sont les points faibles de l'enveloppe. On les rencontre en général lors d'un changement d'une caractéristique (changement de matériau, de géométrie, discontinuité d'isolation...). Dans ce projet, les bâtiments isolés par l'intérieur sont en général munis de rupteurs de ponts thermiques (cf. figure 5) permettant de limiter ces déperditions.
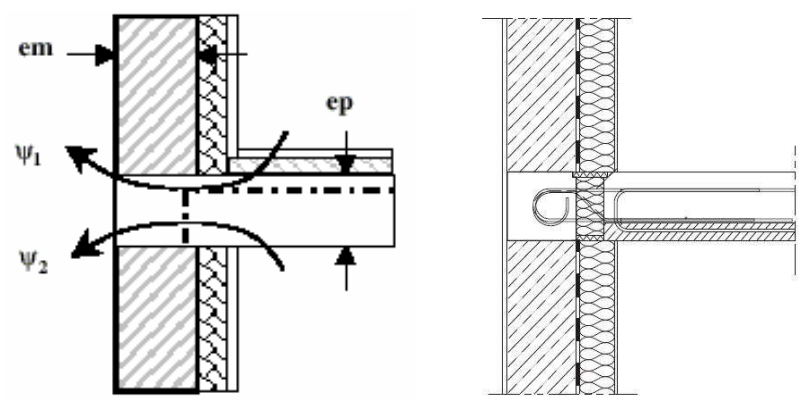

Fig. 5. Liaison mur-plancher intermédiaire dans le cas d'une isolation intérieure (avec un rupteur de pont thermique dans le cas de droite).

L'isolation par l'extérieur choisie pour certains bâtiments présente l'intérêt de réduire considérablement les ponts thermiques (cf figure 6), notamment au niveau des liaisons mur-refend et des planchers intermédiaires.

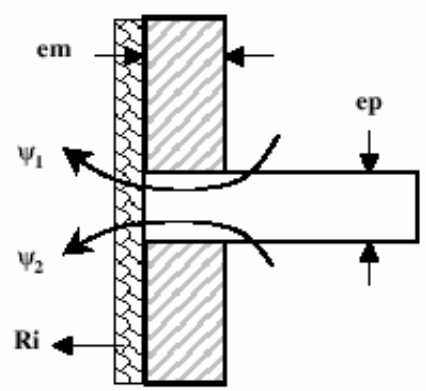

Fig. 6. Liaison mur-plancher intermédiaire le cas d'une isolation extérieure.

Ce type d'isolation permettra également de profiter de l'inertie du bâtiment. L'hiver, les éléments lourds situés dans le volume chauffé participeront au chauffage du bâtiment en restituant l'énergie captée (issue des apports solaires et du chauffage). L'été, ils éviteront les surchauffes en stockant progressivement la chaleur pendant la journée. Il sera 
intéressant de favoriser une ventilation nocturne pour permettre de d'évacuer cette chaleur (cf. figure 7). Dans ces deux cas, l'inertie permet d'améliorer le confort thermique des habitants.

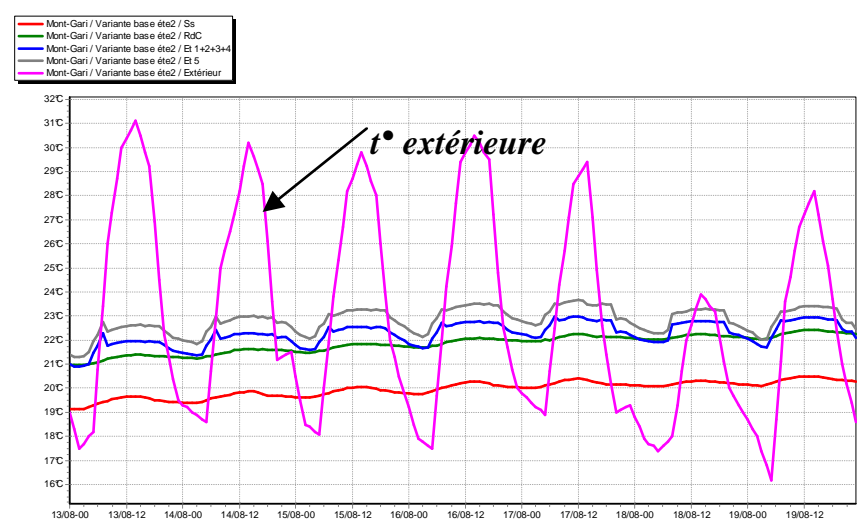

Fig. 7. Température extérieure et températures des différentes zones d'un bâtiment à forte inertie (couplée à une ventilation nocturne) pendant la semaine la plus chaude de l'année.

Les bâtiments doivent présenter un système de ventilation pour apporter de l'air neuf et non pollué à l'intérieur. Le système de ventilation permet également d'évacuer les polluants chimiques, les contaminants biologique et les différentes odeurs afin d'assurer une hygiène de vie acceptable pour les occupants. Une construction doit donc présenter une ventilation qui entraînera des déperditions thermiques importantes par renouvellement d'air.

Dans le cas d'un bâtiment standard, on mettra en œuvre une Ventilation Mécanique Contrôlée (VMC) simple flux qui permet d'extraire l'air suivant les débits réglementaires. Le projet présente ici deux types de VMC plus performantes sur le plan énergétique. D'une part le système de VMC simple flux hygroréglable : l'air est extrait du bâtiment en fonction du taux d'humidité. Ce système permet de réduire d'environ $20 \%$ les déperditions par renouvellement d'air. Le projet présente aussi le système de VMC double flux, le plus performant. L'air extrait sert, à travers un échangeur, à réchauffer l'air entrant. On pourra récupérer jusqu'à $80 \%$ de la chaleur dans le cas de systèmes très performants.

Dans le projet, la fourniture d'énergie viendra en grande partie des énergies renouvelables. $40 \%$ des besoins en énergie pour l'Eau Chaude Sanitaire seront issus de l'énergie solaire thermique grâce aux $1100 \mathrm{~m}^{2}$ de capteurs installés en toiture. Chaque îlot disposera de sa chaufferie bois permettant de prendre en charge $40 \%$ des besoins en énergie pour l'ECS et $80 \%$ pour le chauffage. Enfin, l'électricité issue des capteurs solaires photovoltaïques permettra de couvrir $50 \%$ des besoins des espaces communs.

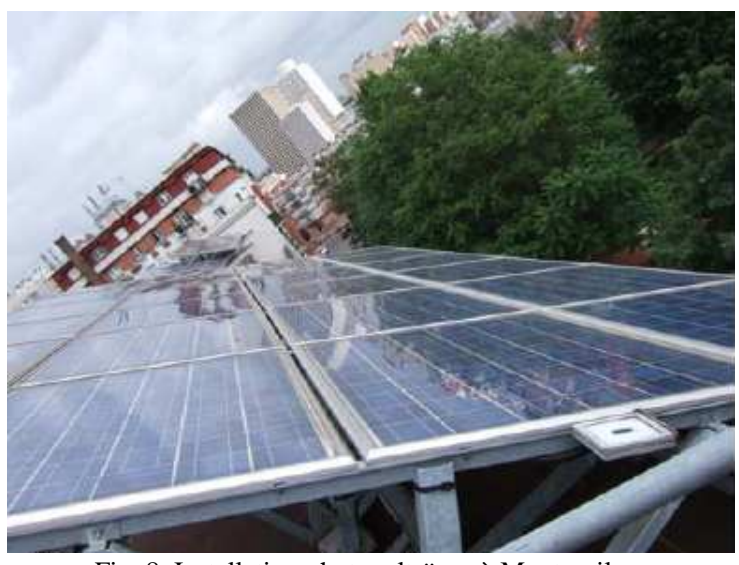

Fig. 8. Installation photovoltaïque à Montreuil

\section{Etude thermique}

Les vingt bâtiments constituant le quartier ont été modélisés en considérant plusieurs zones thermiques, et en prenant en compte les masques générés par les bâtiments voisins. Les figures 9, 10 et 11 montrent la modélisation de quelques bâtiments sous ALCYONE.

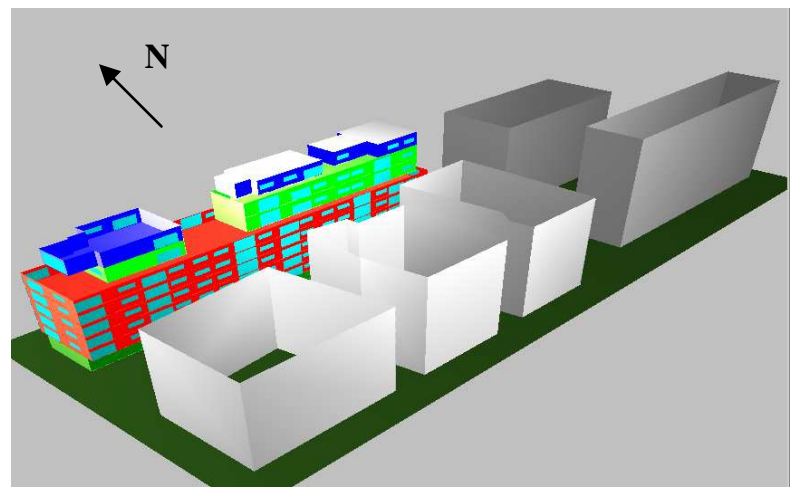

Fig. 9. Ilot A, partie nord (les couleurs correspondent aux différentes zones thermiques du bâtiment D)

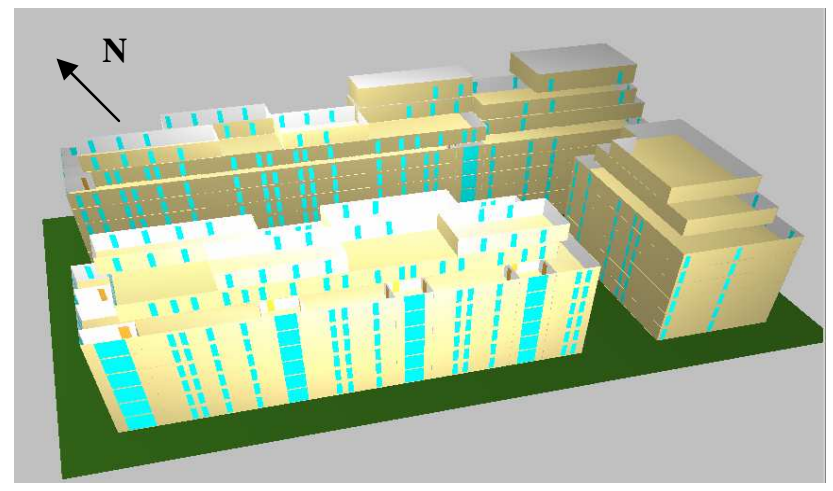

Fig. 10. Ilot A, partie sud, bâtiments QN1

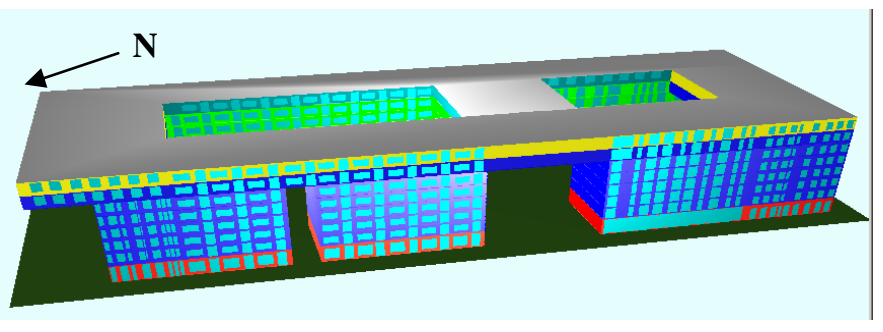

Fig. 11. Ilot C, coté logement 


\begin{tabular}{|c|c|c|c|}
\hline & Standard & Base & Meilleures Pratiques \\
\hline $\begin{array}{l}\text { Epaisseur d'isolant dans les } \\
\text { murs extérieurs }\end{array}$ & $10 \mathrm{~cm}$ & $\begin{array}{l}12(\mathrm{~A}) ; 20(\mathrm{~B}) \& 17(\mathrm{C}) \\
\mathrm{cm}\end{array}$ & $15 \mathrm{~cm}$ \\
\hline Ventilation & Simple Flux $(0,6 \mathrm{vol} / \mathrm{h})$ & $\begin{array}{l}\text { Simple Flux Hygro- } \\
\text { réglable ou Double Flux }\end{array}$ & $\begin{array}{l}\text { Double Flux }(0,6 \text { vol/ } \\
\text { h, efficacité de } 0,7)\end{array}$ \\
\hline Ponts thermiques & $\begin{array}{l}0,7 \mathrm{~W} / \mathrm{mK} \text { pour tous les } \\
\text { éléments }\end{array}$ & $\begin{array}{l}0,2 \mathrm{~W} / \mathrm{mK} \text { pour tous les } \\
\text { éléments }\end{array}$ & $\begin{array}{l}0,5 \mathrm{~W} / \mathrm{mK} \text { pour le } \\
\text { plancher bas }\end{array}$ \\
\hline$U$ moyen des vitrages & $\begin{array}{l}1,8 \mathrm{~W} / \mathrm{m}^{2} \mathrm{~K} \text { (logements) } \\
2,1 \mathrm{~W} / \mathrm{m}^{2} \mathrm{~K} \text { (bureaux) }\end{array}$ & $1,5 \mathrm{~W} / \mathrm{m}^{2} \mathrm{~K}$ & $\begin{array}{l}0,7 \mathrm{~W} / \mathrm{m}^{2} \mathrm{~K} \text { (triple } \\
\text { vitrage) }\end{array}$ \\
\hline
\end{tabular}

TABLEAU 2 : PRINCIPALES CARACTERISTIQUES THERMIQUES DES VARIANTES COMPAREES

Les hypothèses communes à toutes les variantes sont la consigne de température de $20^{\circ} \mathrm{C}$ constante; les apports internes de $21 \mathrm{kWh} / \mathrm{m}^{2}\left(25 \mathrm{kWh} / \mathrm{m}^{2}\right.$ auxquels on retranche les consommations des lave-linge et lave-vaisselle, l'eau chauffée étant évacuée) ; l'occupation des résidents : $25 \%$ d'occupation entre $8 \mathrm{~h} 00$ et $18 \mathrm{~h} 00$ la semaine et $100 \%$ pour le reste du temps (avec $90 \mathrm{~W}$ par résident).

Les trois variantes diffèrent par les techniques (isolation, ventilation...), mais l'architecture influence également les performances : à caractéristiques techniques identiques, la consommation calculée par simulation varie du simple au triple entre les bâtiments 1 et 10 de l'îlot B pour la variante Meilleures Pratiques (ratio de $4 \mathrm{kWh} / \mathrm{m}^{2}$ pour le bâtiment 10 contre $13 \mathrm{kWh} / \mathrm{m}^{2}$ pour le bâtiment 1 ) et quasiment du simple au double $\left(29 \mathrm{kWh} / \mathrm{m}^{2}\right.$ et $\left.58 \mathrm{kWh} / \mathrm{m}^{2}\right)$ dans la variante Standard.

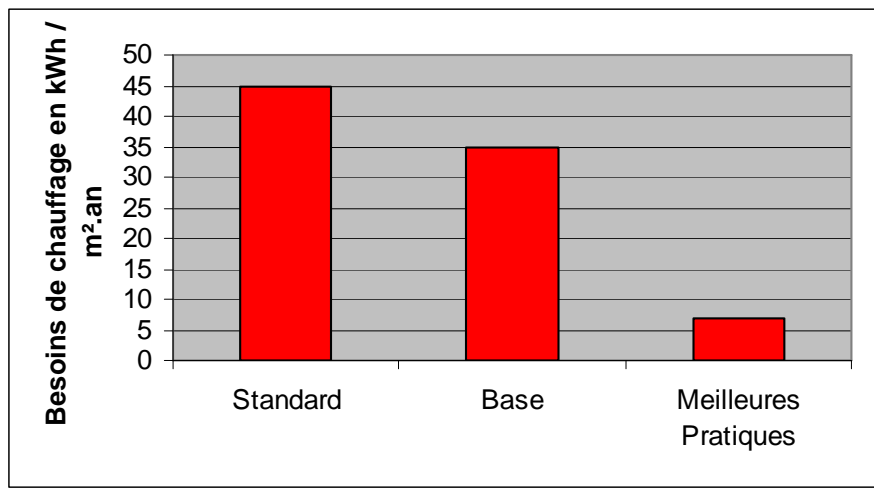

Figure 11 : Besoins de chauffage pour les 3 variantes

Le cahier des charges, répondant aux exigences du programme européen Concerto, limite la consommation de chauffage à $60 \mathrm{kWh} / \mathrm{m}^{2}$ pour les logements et 40 pour les bureaux, soit une moyenne de $56 \mathrm{kWh} / \mathrm{m}^{2}$. Les résultats obtenus avec le logiciel COMFIE correspondent à cet objectif (en moyenne sur les 3 îlots), en considérant un rendement moyen de $60 \%$ pour la chaudière bois.

\section{Analyse de Cycle de Vie}

Les bâtiments sont chauffés à $100 \%$ au gaz dans la variante Standard, à $80 \%$ bois et $20 \%$ gaz dans les variantes Base et Meilleures Pratiques. La consommation d'électricité (78\% nucléaire, $14 \%$ hydroélectrique, $4 \%$ gaz et $4 \%$ charbon) correspond aux données du tableau suivant (sur une base de $85 \mathrm{~m}^{2}$ par logement). Dans les variantes Base et Meilleures Pratiques, 50\% de l'électricité est produite par les panneaux solaires photovoltaïques et une réduction de $40 \%$ de la consommation d'eau est considérée. Enfin les systèmes d'eau chaude sanitaire solaire assurent $40 \%$ des besoins.

\begin{tabular}{cccc}
\hline & Standard & Base & Meilleures Pratiques \\
\hline Consommation électrique & $3000 \mathrm{kWh} /$ logement / an & $2125 \mathrm{kWh} /$ logement / an & $1800 \mathrm{kWh} /$ logement / an \\
Consommation d'eau chaude & $401 /$ résident / jour & $14,41 /$ résident / jour & $14,41 /$ résident / jour \\
Consommation d'eau froide & $1001 /$ résident/ jour & $69,91 /$ résident / jour & $69,91 /$ résident / jour \\
\hline
\end{tabular}

TABLEAU 3 : HyPOTHESES DE L'ANALYSE DE CYCLE DE VIE DES BATIMENTS

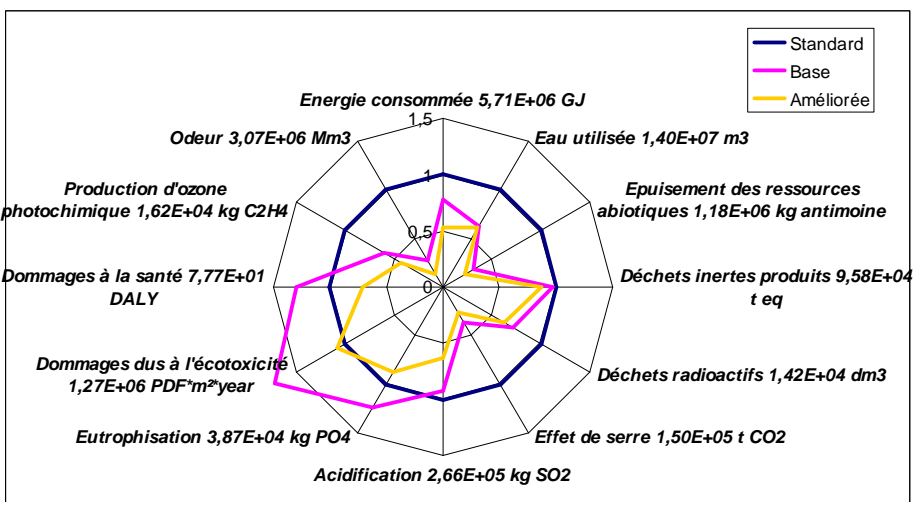

Figure 12 : Analyse de cycle de vie comparative des 3 variantes (logiciel EQUER)

Les impacts environnementaux du projet (base) sont nettement réduits par rapport à la référence Standard sauf en ce qui concerne les dommages dus à l'écotoxicité et les dommages à la santé, ce qui est lié au chauffage au bois. Mais l'inventaire utilisé pour une chaudière bois est issu de la base EcoInvent 1.2, qui reflète la moyenne suisse. Disposer d'un inventaire spécifique aux chaudières mises en œuvre à Lyon améliorerait la précision de cette analyse.

Enfin, le réchauffement climatique étant une problématique majeure du projet Concerto, il est utile d'indiquer les émissions moyennes des espaces publics et des bâtiments (cf. tableau 5).

\begin{tabular}{|c|c|c|c|}
\hline \multicolumn{4}{|c|}{ Emissions de gaz à effet de serre en $\mathrm{kg} \mathrm{CO}_{2}$ éq. / (m².an) } \\
\hline Variante: & Standard & Base & Meilleures Pratiques \\
\hline Bâtiments & 21,9 & 7,9 & 5,6 \\
\hline
\end{tabular}

TABLEAU 5 : EMISSIONS DE GAZ A EFFET DE SERRE DES BATIMENTS ET DES ESPACES PUBLICS

Grâce à leurs performances thermiques élevées et à une forte intégration des énergies renouvelables, les bâtiments sont $64 \%$ moins émetteurs de $\mathrm{CO} 2$ que des bâtiments standards.

\section{CONCLUSION}

La démarche appliquée ici permet de mettre en évidence l'influence de certains choix techniques (architecture, isolation, système de ventilation...) sur les consommations énergétiques d'un bâtiment. Elle permet aussi de montrer les conséquences environnementales des choix du projet Concerto. En plus d'une optimisation énergétique, ce dernier propose de prendre en charge une partie des besoins par les énergies renouvelables.

Cette Analyse de Cycle de vie a été mise en œuvre par les logiciels ALCYONE, COMFIE et EQUER. Le logiciel 
ARIADNE [4] permettra d'appliquer cette approche à l'échelle du quartier (bâtiments et espaces publics).

\section{REFERENCES}

[1] Boissieu C. (2006) "Division par quatre des gaz à effet de serre de la France à l'horizon 2050', Ministère de l'Economie, des Finances et de l'industrie, $\mathrm{p} 14$.

[2] Orselli J. (2005) "Recherche et développement sur les économies d'énergie et les substitutions entre énergies dans les bâtiments", Conseil Général des Ponts et Chaussée, p 10.

[3] Peuportier B., Kellenberger D., Anink D., Mötzl H., Anderson A., Vares S., Chevalier J., and König H. (2004) "Inter-comparison and benchmarking of LCA-based environmental assessment and design tools", Sustainable Building 2004 Conference, Warsaw, paper $\mathrm{n}^{\circ} 75$, 5p.W.-K. Chen, Linear Networks and Systems (Book style). Belmont, CA: Wadsworth, 1993, pp. 123-135.

[4] Popovici E. (2006), Contribution à l'analyse de cycle de vie des quartiers, thèse de doctorat, Ecole des Mines de Paris, 209 p.

[5] ISO, International Organisation for Standardisation (1997) "14040 Environmental Management - Life Cycle Assessment - Principles and Framework", EN ISO 14040, 11p.

[6] Frischknecht R., Jungbluth N., Althaus H.-J., Doka G., Heck T., Hellweg S., Hischier R., Nemecek T., Rebitzer G., Spielmann M. (2004) "Overview and Methodology", ecoinvent report No. 1, Swiss Centre for Life Cycle Inventories, Dübendorf, Suisse, 75 p.

[7] Houghton J.T., Ding Y., Griggs D.J., Nogger M., van der Linden P.J., Dai X., Maskel K., Johnson C.A. (2001), Intergovernmental panel on Climate Change (IPCC), Climate change 2001 : the scientific basis, Cambridge University Press, $881 \mathrm{p}$.

[8] Guinée J. B., (final editor), Gorrée M., Heijungs R., Huppes G., Kleijn R., de Koning A., van Oers L., Wegener Sleeswijk A., Suh S., Udo de Haes H. A., de Bruijn H., van Duin R., Huijbregts M. A. J., Lindeijer E., Roorda A. A. H., Weidema B. P. (2001) "Life cycle assessment; An operational guide to the ISO standards", Ministry of Housing,
Spatial Planning and Environment (VROM) and Centre of Environmental Science (CML), Den Haag and Leiden, Pays Bas, 704 p.

[9] Goedkoop M. et Spriensma R. (2000) "The Eco-indicator 99 : A damage oriented method for life cycle impact assessment", PRé Consultants, Amersfoort, Pays Bas, 142 p.

[10] Salomon T., Mikolasek R. et Peuportier B. (2005), Outil de simulation thermique du bâtiment, COMFIE, Journée SFT-IBPSA « Outils de simulation thermo-aéraulique du bâtiment », La Rochelle, 8p.

[11] Peuportier B. et Blanc Sommereux I. (1990) "Simulation tool with its expert interface for the thermal design of multizone buildings", International Journal of solar Energy, vol. 8, pp 109-120Letter Symbols for Quantities, ANSI Standard Y10.5-1968.

[12] Polster B., Peuportier B., Blanc Sommereux I., Diaz Pedegral P., Gobin C., Durand E. (1996) "Evaluation of the environmental quality of buildings - a step towards a more environmentally conscious design”, Solar Energy, vol. 57 no3, pp 219-230.

\section{POUR ALLER PLUS LOIN DANS LA THERMIQUE DES BATIMENTS}

Sacadura J. (1980) "Initiation aux transferts thermiques", Ed. Technique et Documentation.

(1997) "Aide-mémoire du thermicien", Elsevier.

Salomon T., Bedel S. (1999) "La maison des négawatts", Ed Terre Vivante.

Salomon T., Bedel S. (2004) 'Fraîcheur sans clim”, Ed Terre Vivante.

Liébard A., De Herde A. (1996-2003) "Guide de l'architecture bioclimatique', 6 tomes, Systèmes Solaires.

Peuportier B. (2003) 'Eco-conception des bâtiments', Presses de l'Ecole des Mines, 276p.

http://retscreen.gc.ca/

http://www.ises.org/

http://www.agores.org/

http://www.cler.org/fr/index.htm

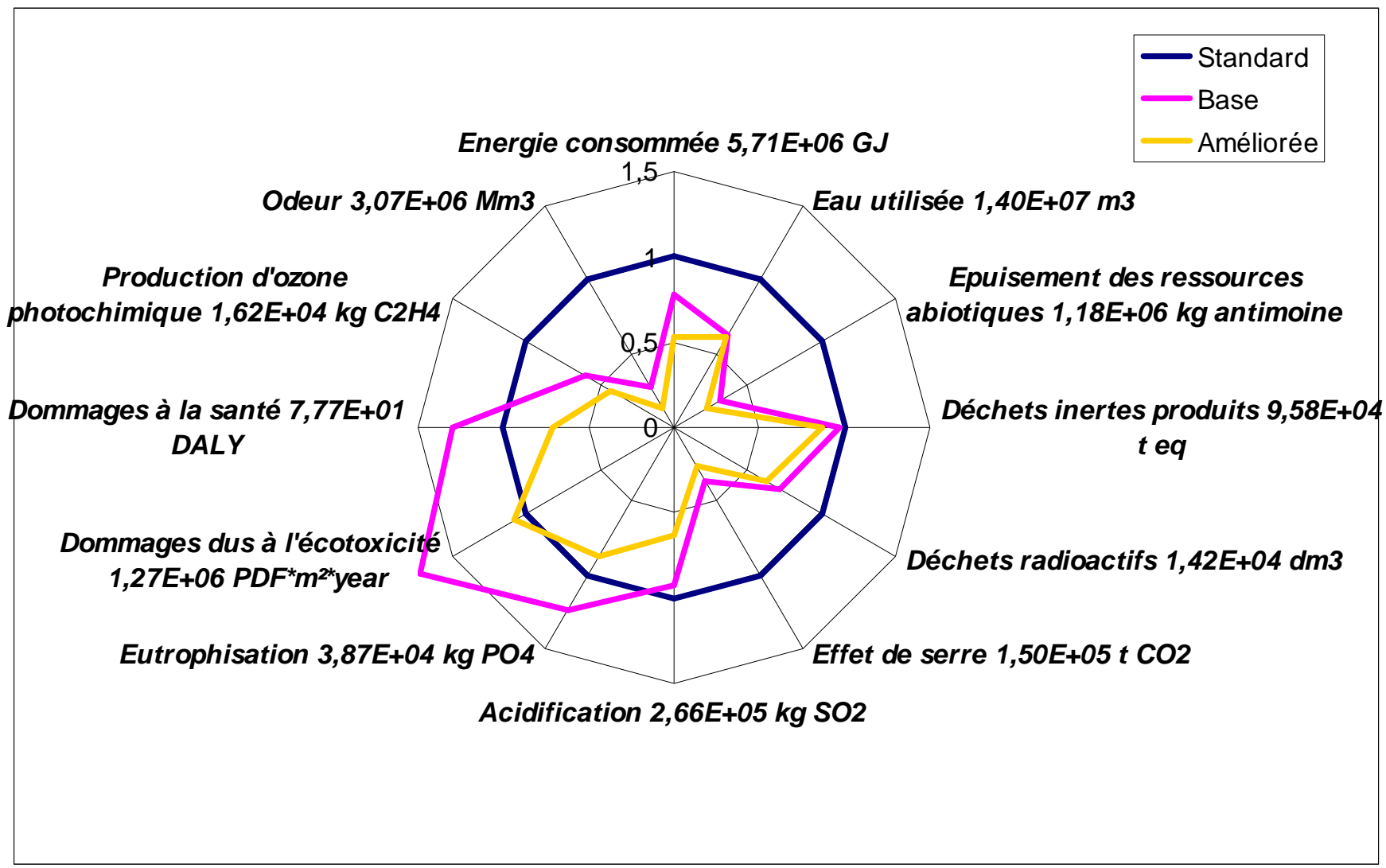

Figure 12 : Analyse de cycle de vie comparative des 3 variantes (logiciel EQUER) 